Article

\title{
Measurement invariance of the Nine-Item Internet Gaming Disorder Scale (IGDS9-SF) across Siri Lanka, Turkey, Aus- tralia, and the USA
}

\author{
Amira Mohammed Ali ${ }^{1 *}$, Rasmieh Al-Amer ${ }^{2}$, Maha Atout ${ }^{3}$, Sawsan Abuhammad ${ }^{4}$, Tazeen Saeed Ali 5 , Ayman M. \\ Hamdan Mansour ${ }^{6}$, Haitham Khatatbeh ${ }^{7}$, Abdulmajeed A. Alkhamees ${ }^{8 *}$ and Amin Omar Hendawy ${ }^{9}$ \\ 1 Department of Psychiatric Nursing and Mental Health, Faculty of Nursing, Alexandria University, Smouha, \\ Alexandria 21527, Egypt \\ 2 Faculty of Nursing, Isra University, Amman, Jordan, r.al-amer@outlook.com \\ 3 School of Nursing, Philadelphia University, Amman 19392, Jordan, m.atout@philadelphia.edu.jo \\ 4 Department of Maternal and Child Health, Faculty of Nursing, Jordan University of Science and Technol- \\ ogy, Irbid 22110, Jordan, Shabuhammad@just.edu.jo \\ 5 School of Nursing and Midwifery, Aga Khan University, 3500, Karachi, Pakistan. tazeen.ali@aku.edu \\ 6 Department of Psychiatric and Mental Health Nursing, School of Nursing, the University of Jordan, Am- \\ man, Jordan, a.mansour@ju.edu.jo \\ 7 Faculty of Health Sciences, Doctoral School of Health Sciences, University of Pécs, 7621 Pécs, Hungary, \\ Khatatbeh.haitham@etk.pte.hu \\ 8 Department of Medicine, College of Medicine and Medical Sciences, Qassim University, Al Qassim, Buray- \\ dah 52571, Saudi Arabia; A.alkhamees@qu.edu.sa \\ 9 Department of Biological Production, Tokyo University of Agriculture and Technology, Tokyo 183-8509, \\ Japan, amin.hendawy@gmail.com \\ * Correspondence: mercy.ofheaven2000@gmail.com(A.M.A.); A.alkhamees@qu.edu.sa (A.A.A.); \\ Tel.: +2-03-4291-578 (A.M.A.); +966-06-3800916 (A.A.A.)
}

\begin{abstract}
The prevalence of internet gaming disorders (IGD) is considerably high among youth, especially with social isolation imposed by the ongoing COVID-19 pandemic. IGD adversely affects mental health, quality of life, and academic performance. The Internet Gaming Disorder Scale (IGDS9-SF) is designed to detect IGD according to DSM-IV diagnostic criteria. However, inconsistent results are reported on its capacity to diagnose IGD evenly across different cultures. To ensure the suitability of the IGDS9-SF as a global measure of IGD, this study examined the psychometric properties of the IGDS9-SF in a sample of Sri Lankan university students $(\mathrm{N}=322$, mean age $=17.2 \pm 0.6$, range $=16-18$ years, $56.5 \%$ males) and evaluated its measurement invariance across samples from Sri Lanka, Turkey, Australia, and the USA. Among Sri Lankan students, a unidimensional structure expressed good fit, invariance across different groups (gender, ethnicity, income, etc.), adequate criterion validity (strong correlation with motives of internet gaming, daily gaming duration, and sleep quality), and good reliability (alpha $=0.81$ ). Males and online multiplayers expressed higher IGD levels, greater time spent gaming, and more endorsement of gaming motives (e.g., Social and Coping) than females and offline players. Across countries, the IGDS9-SF was invariant at the configural, metric, and scalar levels, albeit strict invariance was not maintained. The lowest and highest IGD levels were reported among Turkish and American respondents, respectively. In conclusion, the IGDS9-SF can be reliably used to measure IGD among Sri Lankan youth. Because the scale holds scalar invariance across countries, its scores can be used to compare IGD levels in the studied countries.
\end{abstract}

Keywords: Coronavirus disease 2019; Internet Gaming Disorder Scale9-Short form (IGDS9-SF); university students; factorial structure/psychometric properties/structural validity/validation; cultur*; invariance; gender; game type 
Internet gaming is commonly used as a recreational activity among children, adolescents, and young adults [1]. With the expansion of internet technology, internet gaming has been employed to serve educational purposes, promote physical activity, develop cognitive skills and therapeutic actions (e.g., reasoning, spatial awareness, and problem-solving) [2]. While most industries were negatively affected during coronavirus disease 2019 (COVID-19) pandemic, the gaming industry has considerably flourished worldwide, with increased gaming time by previous users, numbers of new users, female users, and traffic in online mobile gaming. Indeed, the World Health Organization's collaborative campaign (\#PlayApartTogether) has been employed to promote online gaming as a method of fostering socialization while maintaining spatial distancing to prevent infection spread [2-4]. Increased risk for excessive gaming and increased screen use time among youth during COVID-19 may be a method to compensate for negative emotions associated with social isolation/being homebound, lack of meaningful activities (e.g., due to closure of schools and workplaces), inability to use entertainment activities that were available before the pandemic (e.g., clubs, cinemas, etc.), and COVID-19 burnout [3-6].

Children and adolescents are particularly vulnerable to develop maladaptive patterns of excessive or problematic gaming [3]. Internet Gaming Disorder (IGD) is a behavioral pattern of persistent and recurrent involvement in online and offline games, resulting in remarkable distress and impairment in essential life activities (work and study) for a period of 12 months or more [7,8]. The global prevalence of IGD ranges between $0.7 \%$ and $15.6 \%[9,10]$. In a Chinese study comprising 2863 school children, $83.0 \%$ played video games during the COVID-19 pandemic. Excessive and pathological gaming were evident in $20.9 \%$ and $5.3 \%$ of the participants [11].

Factors associated with increased IGD during COVID-19 include male gender, young age, loneliness, lack of parental support and/or supervision, mental health problems, and low socio-economic status [1,3,11,12]. Internet gaming among youth is associated with a plethora of negative consequences: poor health-related quality of life, sleep disturbances, impaired life skills, low self-esteem, concentration problems, poor communication skills, higher social distress, poor real-life relationships, loneliness, aggression, poor academic/work performance, poor impulse control, and tendency toward psychopathology [2,13-16]. Persistent or excessive gaming may increase the risk of more serious mental health problems [4], with suicide reported among adolescents and emerging adults with psychological predispositions who played videogames that take up many hours a day such as PUBG [12].

The DSM-5 classifies IGD in Section III-disorders requiring further investigation $[8,14]$. According to the DSM-5, endorsing five out of nine normative symptomatic criteria is sufficient for establishing IGD as a diagnosis: 1) preoccupation with playing on the internet/degital games; 2) withdrawal symptoms when internet games are not available; 3 ) tolerance noted by increased time spent in gaming; 4) relapse noted by failed attempts to quit gaming; 5) loss of interest in other previous hobbies/entertainment behaviors because of, and with the exception of, online games; 6) continued and excessive use of online games despite knowledge of psychosocial problems it causes; 7) deception of relatives, therapists, or other people about the amount of time spent in gaming; 8) mood modification is noted by use of online games to escape or mitigate negative emotions; and, 9) losing significant interpersonal relationships, work and educational or professional opportunities as a result of participating in internet gaming [7,9]. These nine criteria have been described based on existing research on pathological gambling and substance use disorder, associating IGD measurement with methodological issues [8]. Among 18 measures designed to assess IGD, the Internet Gaming Disorder Scale 9-Short-Form (IGDS9-SF), a brief form of the IGDS, has been designed to detect all nine diagnostic criteria of IGD $[8,16]$.

The IGDS9-SF has been translated into many languages, including Italian and Albanian [8], Korean [15], Spanish [14], Polish [17], Turkish [10], Arabic [18], Portugeese [19], etc. Although numerous studies report good reliability and validity of the IGDS9-SF in 
samples from Western and developed countries, its psychometric evaluation in various cultural contexts is limited, which may strict its use as a global measure of IGD [8,17].

Cultural orientations may considerably influence the way individuals respond to a symptom scale. For instance, collectivistic cultures put a great appreciation for group values and norms while conditions entailing deviation from group norms (e.g., mental disorders such as IGD) can be stigmatized [20]. Therefore, collectivistic individuals tend to express IGD scores close to the mean, resulting in minimal of range of IGDS9-SF item responses. On the other hand, individualistic cultures entail appreciation of individual goals, values, competition and achievement as a base of social hierarchies. Therefore, individualistic persons tend to compete more in gaming to achieve higher rankings $[8,21]$.

Measurement invariance is frequently tested to ensure usability of a measure for comparing the levels of a latent construct across different groups (e.g., cultures, ethnicities, genders, age) [22,23]. Measurement invariance is assessed at four levels: configural, metric, scalar, and strict. Ideally, an invariant scale successfully reflects similar conceptualization of the underlying latent structure among groups (usually by reporting the same observed scores), similar degree of endorsement of items, and a capacity to objectively compare scale mean scores among groups. Comparisons based on non-invariant measures, especially at the scalar level, are likely to be invalid because group scores are confounded by differences in scaling properties across groups [8,22]. Although all previous findings support the unidimensional structure of the IGDS9-SF, some degrees of measurement non-invariance have been expressed especially in studies comprizing English-speaking (e.g., Australia, United Kingdome (UK), and the United States of America (USA)) and non English-speaking samples e.g., Polish and Indian [17,21]. Even among English-speakers from the USA and Australia, investigations of time invariance (three months) uncovered partial metric and scalar non-invariance among Australian gamers [24].

Given the widespread of IGD and related mental health and academic adverse effects among youth, careful identification and proper management of IGD in this group may have implications for preventing/mitigating psychiatric comorbidities [23,25]. Lack of validation of IGD measures in developing countries such as Sri Lanka represents a challenge for IGD detection and treatment. It is not clear if IGD levels among youth from Sri Lanka can be compared with IGD levels among countries with evolving economy such as Turkey and developed countries such as Australia and the USA. These four countries do not only vary according to their economy but also according to the dominant cultural orientations. To fill the gap, the current study aimed to examine the structure, invariance, and criterion validity of the IGD9-SF among university students from Sri Lanka. We hypothesized that the IGD9-SF would express a unidimensional structure that would be invariant across groups of gender, ethnicity, etc. We also expected that the IGD9-SF would strongly correlate with average daily gaming time, different motives of gaming, as well as sleep quality and quantity. In addition, we expected that the IGD9-SF would express some none-invariance across countries (Sri Lanka, Turkey, Australia, and the USA).

\section{Materials and Methods}

\subsection{Study design, participants, and procedure}

This cross-sectional study is a secondary analysis based on three publicly accessible datasets. The first dataset is affiliated with University of Colombo Faculty of Medicine, Sri Lanka [26]; it is associated with a published study [27]. This dataset comprises a sample of advanced level university students obtained through random cluster sampling from four schools of the Colombo Educational Zone in Sri Lanka. This sample was used to examine the psychometric properties of the IGDS9-SF in Sri Lanka [27]. The sociodemographic, academic, and gaming characteristics of this sample are shown in Table 1. 
Table 1. Sociodemographic, academic, and gaming characteristics of university students from Sri Lanka

\begin{tabular}{|c|c|}
\hline Participant characteristics & $(\mathrm{N}=322)$ \\
\hline Age in years mean (SD) & $17.2 \pm 0.6$ \\
\hline \multicolumn{2}{|l|}{ Gender } \\
\hline Males & $182(56.5 \%)$ \\
\hline Females & $140(43.5 \%)$ \\
\hline \multicolumn{2}{|l|}{ Ethnicity } \\
\hline Sinhala & $249(77.3 \%)$ \\
\hline Others & $73(22.7 \%)$ \\
\hline \multicolumn{2}{|l|}{ Language used } \\
\hline Sinhala & $152(47.2 \%)$ \\
\hline English & $170(52.8 \%)$ \\
\hline \multicolumn{2}{|l|}{ Major } \\
\hline Physical education & $88(27.3 \%)$ \\
\hline Commerce & $106(32.9 \%)$ \\
\hline Biology & $67(20.8 \%)$ \\
\hline Arts & $51(15.9 \%)$ \\
\hline Others & $10(3.1 \%)$ \\
\hline \multicolumn{2}{|l|}{ Monthly income (SLR) $\boldsymbol{\Delta}$} \\
\hline$<100,000$ & $124(38.5 \%)$ \\
\hline$<200,000$ & $106(32.9 \%)$ \\
\hline$>200,000$ & $92(28.6 \%)$ \\
\hline \multicolumn{2}{|l|}{ Gaming Type } \\
\hline Offline single player & $109(33.9)$ \\
\hline Online multiplayer & $164(50.9)$ \\
\hline Online single player & $49(15.2)$ \\
\hline \multicolumn{2}{|l|}{ Gaming hours/day } \\
\hline One hour or less & $172(53.4)$ \\
\hline Two to three hours & $91(28.3)$ \\
\hline More than three hours & $59(18.3)$ \\
\hline \multicolumn{2}{|l|}{ Device } \\
\hline Mobile & $206(64.0 \%)$ \\
\hline Others & $116(36.0 \%)$ \\
\hline \multicolumn{2}{|l|}{ Sleep hours/day } \\
\hline Five hours or less & $102(31.7 \%)$ \\
\hline Six hours or more & $220(68.3 .2 \%)$ \\
\hline
\end{tabular}

Invariance of the IGDS9-SF across countries is based on data of the IGDS9-SF only from an international sample, which was integrated from three publicly accessible datasets, including the current sample from Sri Lanka [26]. It also included a sample from Turkey comprising 244 university students who reported playing digital games. Males and females were almost equally represented $(n=113,46.3 \%)$ and $(n=131,53.7 \%)$, respectively [28]. No further details are available about the characteristics of those students. The third dataset comprised online gamers from Australia $(\mathrm{N}=738$, mean age $=25.8 \pm 7.6$, range $=18-72$ years, $49.3 \%$ females, $71.7 \%$ employed, $32.7 \%$ students $)$ and the USA $(\mathrm{N}=$ 222 , mean age $=27.0 \pm 8.0$, range $=18-63$ years, $54.1 \%$ females, $70.3 \%$ employed, $39.2 \%$ students) [29]. Data collection was obtained through an anonymous online survey conducted through SurveyGizmo, and an ethical approval for data collection was issued by the ethics committee of Cairnmillar Institute. Further details on the characteristics of the 
participants from those countries are reported in detail elsewhere [30]. Because all the datasets are shared under the terms of creative common license (CC BY 4.0) [26,28] or are in the public domain [29], we did not obtain an ethical approval for the current study.

\subsection{Data collection measures}

The questionnaire addressed to students from Sri Lanka comprised a Personal Information Form inquiring about students' age, gender, major, ethnicity, gaming experience (hours, type of gaming, age of start), etc. It also comprised other measures, including the Internet Gaming Disorder Scale 9-Short Form (IGDS9-SF), a brief measure of the severity of IGD symptoms [31]. The scale consists of nine items. Each item is rated on a 5-point Likert scale ranging from $(1=$ never $)$ to $(5=$ very often). The maximum and minimum scores of the IGDS9-SF are 9 and 45. Higher scores reflect higher levels of problematic internet gaming [31]. The IGDS9-SF was administered both in Sinhala and English. Its reliability in this Sri Lankan sample is good (coefficient alpha $=0.81$ ).

Motives for Online Gaming Questionnaire (MOGQ), a scale that comprises 27 items, was developed by Demetrovics et al to measure seven major gaming motives: Social (building and maintaining social relationships), Escape (escaping from reality), Coping (coping with stress and distress), Competition (challenging and competing with others), Skill Development (attention and coordination), Fantasy (in-game identities and experience), and Recreation (entertainment and enjoyment). Items are rated on a five-point Likert scale $(1=$ almost never/never $)$ to $(5=$ almost always/always) [32]. Its reliability in the current sample is excellent (coefficient alpha $=0.92$ ).

The Single Item Sleep Quality Scale (SISQ) was used to evaluate sleep quality. The response is rated on a scale from 1 to 10 , with higher scores indicating better sleep quality [33].

Self-esteem has been assessed by a single question prompting the participants to rate their self-esteem on a 5 -point Likert $(1=$ very poor $)$ to $(5=$ excellent $)$.

\subsection{Statistical analysis}

Checking the original Sri Lankan dataset, which comprised 395 responses, for missing data revealed that seventy-three responses had missing data on the IGDS9-SF. Therefore, they were excluded from the analysis, ending with a final sample of 322 respondents - response rate $=82 \%$. The distribution of the IGDS9-SF and MOGQ was examined using Shapiro-Wilk test. Quantitative variables with normal distribution were described by mean and standard deviation while those with non-normal distribution were described using median and interquartile range (IQR: 25\%-75\%). Categorical variables were described using number and percentage.

Based on the literature, the unidimensional structure of the IGDS9-SF was examined in the Sri Lankan sample by confirmatory factor analysis (CFA) using maximum likelihood method of estimation with bootstrap that generates 2000 random replications. Multigroup CFA was conducted to examine invariance of the IGDS9-SF across countries (Sri Lanka, Turkey, Australia, and the USA) in the international sample as well as across groups of gender, ethnicity, language used to complete the questionnaire, income, device, game type, and academic major in the Sri Lankan sample (groups are shown in Table 1).

Chi square $(\chi 2)$ index is largely dependent on sample size, and well-fitting models with minor misspecifications may be disqualified based on a significant $\chi 2$ [25]. Meanwhile, absolute fit indices represent more reliable indicators of model fit because they are sample-size independent. Therefore, we considered model fit in CFA/multigroup CFA to be good or acceptable based on Comparative Fit Index (CFI) and Tucker-Lewis Index (TLI) equal to or above 0.95 and 0.90 , respectively, along with root mean square error of approximation (RMSEA) and standardized root-mean-square residual (SRMR) less than 0.06 and 0.08 , respectively [34]. 
The internal consistency of the IGDS9-SF was examined by coefficient alpha, alphaif-item deleted, and item-total correlations. Its criterion validity was evaluated by Spearman's r correlations with the MOGQ, number of sleeping hours, SISQ, and single-item measure of self-esteem in the Sri Lankan sample. Additionally, Mann Whitney $U$ test and Kruskal Wallis test were used to examine differences in the number of gaming hours as well as key constructs measured by MOGQ among groups of gender and game type since differences in IGD were depicted in these groups, and they expressed a tendency toward non-invariance in multigroup CFA. Statistical analyses were conducted in SPSS and Amos, and significance was considered at a probability level less than 0.05 in two-tailed tests.

\section{Results}

\subsection{Confirmatory factor analysis and invariance analysis of the Internet Gaming Disorder Scale} 9-Short Form

As shown in Table 2, the IGDS9-SF expressed excellent fit among students from Sri Lanka. According to Supplementary Table 1, the scale expressed invariance at the configural, metric, and scalar levels among Sri Lankan students across groups of gender, ethnicity, language used to complete the questionnaire, income, game type, academic major, and device used for gaming. However, there was a tendency toward configural non-invariance across groups of monthly income $(\Delta \mathrm{CFI}=0.022, \Delta \mathrm{TLI}=0.020)$ and strict noninvariance across groups of gender and game type $(\Delta C F I=0.042,0.028 ; \Delta T L I=0.037$, 0.020). However, $\triangle$ RMSEA was within the acceptable range in all tests, which supports invariance of the IGDS9-SF.

The IGDS9-SF expressed good fit in the Turkish, Australian, and American subsamples as well (Table 2). It was invariant at the configural, metric and scalar levels across participants from the four countries. However, strict invariance was not maintained $(\Delta C F I$ $=0.147, \Delta \mathrm{TLI}=0.120, \Delta \mathrm{RMSEA}=0.027)$ due to minor misspecifications in the covariances of few items among participants from Turkey, Australia, and the USA (Figure 1). Kruskal Wallis test revealed significant differences in the levels of IGD across countries $(\mathrm{H}(3)=$ 185.99, $\mathrm{p}=0.001$ ), with the lowest levels reported in participants from Turkey (median $(\mathrm{IQR})=13.0(10.0-18.0))$ and Sri Lanka (median $(\mathrm{IQR})=18.0(13.0-22.0))$ while the highest levels were reported in participants from the USA (median $(\mathrm{IQR})=21.0(17.0-27.0))$ and Australia (median $(I Q R)=20.0$ (16.0-25.0)). Shapiro Wilk W test showed that the IGDS9SF demonstrates a similar distribution in all the samples. Reliability tests showed that the internal consistency of the scale in the international subsamples ranged between very good and excellent (Table 2).

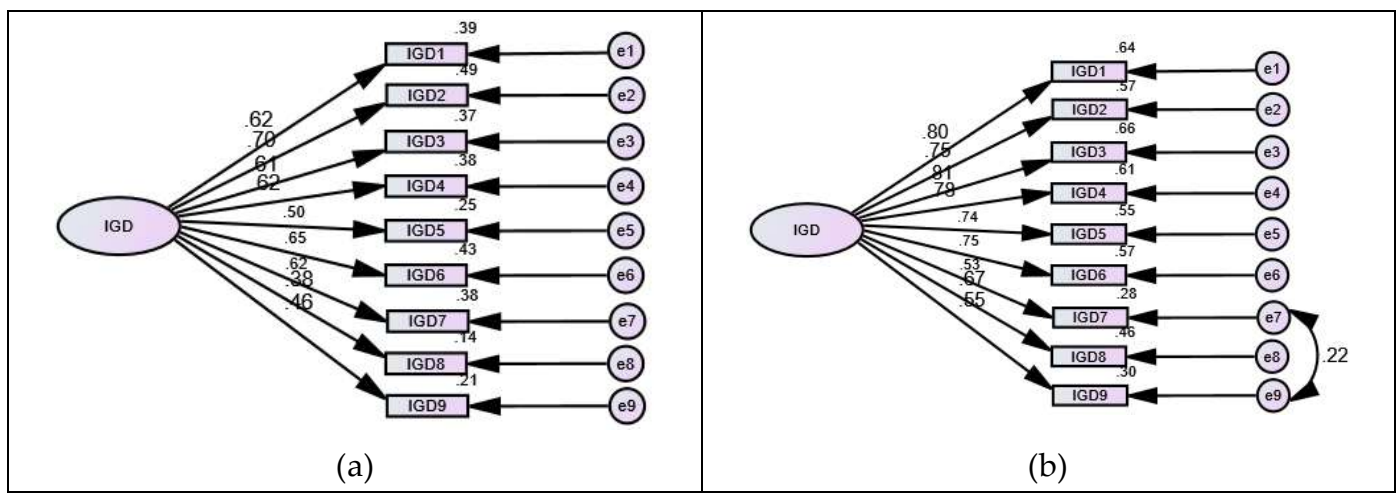




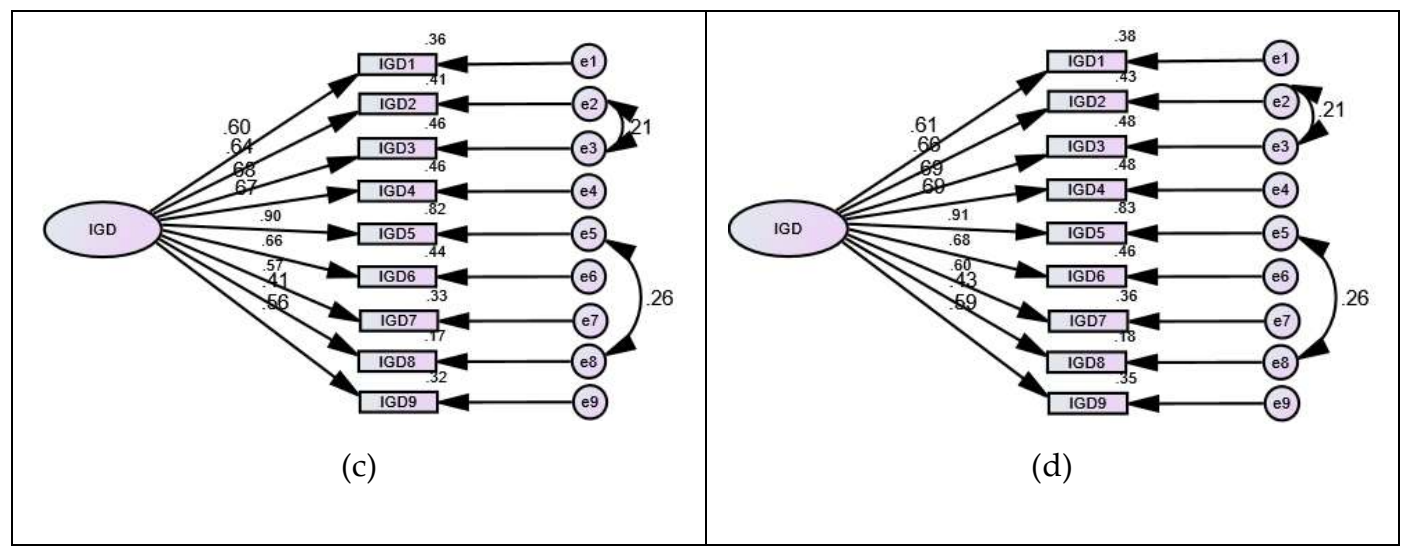

Figure 1. Factor structure of the Internet Gaming Disorder9-Short Form (IGDS9-SF) among participants from Sri Lanka (a), Turkey (b), Australia (c), and the USA (d). 
Table 2. Goodness-of-fit indices for the one-factor structure of the Internet Gaming Disorder Scale 9-Short form (IGDS9-SF) among university students from Sri Lanka, its invariance across countries, normality tests, and internal consistency

\begin{tabular}{|c|c|c|c|c|c|c|c|c|c|c|c|c|c|c|c|c|}
\hline Groups & Invariance levels & $\chi^{2}$ & df & $P$ & $\Delta \chi^{2}$ & $\Delta \mathrm{df}$ & $p\left(\Delta \chi^{2}\right)$ & CFI & $\Delta \mathrm{CFI}$ & TLI & $\Delta \mathrm{TLI}$ & RMSEA & $\triangle$ RMSEA & SRMR & WA & Coefficient alpha \\
\hline \multirow[t]{9}{*}{ Countries } & Sri Lanka & 41.846 & \multirow{2}{*}{27} & \multirow{2}{*}{0.034} & & & & \multirow{2}{*}{0.978} & & \multirow{2}{*}{0.970} & & \multirow{2}{*}{0.041} & & 0.0357 & 0.961 & 0.811 \\
\hline & Turkey & 73.680 & & & & & & & & & & & & 0.0353 & 0.838 & 0.902 \\
\hline & Australia & 109.946 & 26 & 0.001 & & & & 0.967 & & 0.954 & & 0.076 & & 0.0359 & 0.950 & 0.862 \\
\hline & USA & 113016 & 25 & 0.001 & & & & 0.965 & & 0.950 & & 0.068 & & 0.0350 & \multirow{2}{*}{0.965} & \multirow{3}{*}{0.876} \\
\hline & USA & 113.010 & 25 & 0.001 & & & & 0.967 & & 0.953 & & 0.069 & & 0.0350 & & \\
\hline & Contigural & 351.228 & \multirow{2}{*}{108} & \multirow{2}{*}{0.001} & \multirow{2}{*}{108.578} & \multirow{2}{*}{24} & \multirow{2}{*}{0.001} & \multirow{2}{*}{0.956} & & \multirow{2}{*}{0.941} & & \multirow[t]{2}{*}{0.038} & & $0.035 \%$ & & \\
\hline & Metric & 459.806 & & & & & & & 0.016 & & 0.006 & & -0.002 & 0.0577 & & \\
\hline & Strong & 473.968 & $\begin{array}{l}132 \\
135\end{array}$ & 0.001 & 14.163 & 3 & 0.003 & 0.938 & 0.002 & & 0.001 & \multirow{2}{*}{0.041} & -0.001 & 0.0735 & & \\
\hline & Strict & 1307.42 & $\begin{array}{l}135 \\
162\end{array}$ & $\begin{array}{l}0.001 \\
0.001\end{array}$ & 833.458 & 27 & 0.001 & $\begin{array}{l}0.938 \\
0.791\end{array}$ & 0.147 & $\begin{array}{l}0.934 \\
0.814\end{array}$ & 0.120 & & 0.027 & 0.1086 & & \\
\hline
\end{tabular}

$\chi^{2}$ : chi-square; df: degrees of freedom; CFI: comparative fit index; TLI: Tucker-Lewis index; RMSEA: root mean square error of approximation; CI: confidence interval; SRMR: standardized root mean residual; $\triangle$ : Shapiro-Wilk $W$ test with all $p$ values $<0.01$; values in boldface indicate variance. 


\begin{tabular}{|c|c|c|c|c|c|c|c|c|c|c|c|c|}
\hline Variables & 1 & 2 & 3 & 4 & 5 & 6 & 7 & 8 & 9 & 10 & 11 & 12 \\
\hline 1. IGDS9-SF & -- & & & & & & & & & & & \\
\hline 2. Gender & $0.235^{* *}$ & -- & & & & & & & & & & \\
\hline 3. Ethnicity & $0.131^{*}$ & $0.288^{* *}$ & -- & & & & & & & & & \\
\hline 4. Sleep quality & $-0.120^{*}$ & 0.082 & 0.000 & -- & & & & & & & & \\
\hline 5. Gaming hours/day & $0.532^{* *}$ & $0.312^{* *}$ & $0.170^{* *}$ & -0.066 & -- & & & & & & & \\
\hline 6. Self-esteem & -0.104 & 0.084 & 0.030 & $0.193 * *$ & -.008 & -- & & & & & & \\
\hline 7. Social & $0.514 * *$ & $0.204 * *$ & -0.014 & -0.038 & $0.463 * *$ & 0.033 & -- & & & & & \\
\hline 8. Escape & $0.616 * *$ & $0.123 *$ & 0.043 & $-0.161 * *$ & $0.383 * *$ & $-0.122 *$ & $0.537 * *$ & -- & & & & \\
\hline 9. Competition & $0.367 * *$ & 0.085 & 0.028 & -0.059 & $0.340 * *$ & 0.017 & $0.495 * *$ & $0.452 * *$ & -- & & & \\
\hline 10. Coping & $0.513 * *$ & $0.119 *$ & -0.042 & $-0.134 *$ & $0.295 * *$ & $-0.149 * *$ & $0.503 * *$ & $0.708 * *$ & $0.426 * *$ & - & & \\
\hline 11. Skill development & $0.398 * *$ & 0.060 & -0.098 & -0.030 & $0.334 * *$ & -0.057 & $0.552 * *$ & $0.538 * *$ & $0.534 * *$ & $0.590 * *$ & -- & \\
\hline 12. Fantasy & $0.451 * *$ & $0.145 * *$ & 0.073 & -0.074 & $0.321 * *$ & -0.017 & $0.469 * *$ & $0.604 * *$ & $0.459 * *$ & $0.505 * *$ & $0.497 * *$ & -- \\
\hline 13. Recreation & $0.389 * *$ & $0.295 * *$ & 0.098 & 0.020 & $0.448 * *$ & -0.024 & $0.464 * *$ & $0.254 * *$ & $0.356 * *$ & $0.439 * *$ & $0.418 * *$ & $0.322 * *$ \\
\hline Median & 18.0 & -- & -- & 7.0 & -- & 4.0 & 8.0 & 7.0 & 9.0 & 9.0 & 8.0 & 7.0 \\
\hline IQR (Q1-Q3) & $13.0-22.0$ & -- & -- & $5.0-8.0$ & -- & $3.0-4.0$ & $5.0-8.0$ & $5.0-10.0$ & $6.0-12.0$ & $7.0-12.0$ & $5.8-12.0$ & $4.0-10.0$ \\
\hline
\end{tabular}




\subsection{Reliability and criterion validity of the Internet Gaming Disorder Short-Form 9}

Among Sri Lankan students, the IGDS9-SF expressed adequate internal consistency (coefficient alpha $=0.81$ ), item-total correlations ranging between 0.341 and 0.611 , and alpha if item deleted ranging between 0.779 and 0.816 . It also demonstrated adequate criterion validity by exhibiting strong positive correlation with all motives of gaming measured by the MOGQ; the highest correlations were demonstrated with the dimensions of Escape and Coping (Table 3). It also correlated with the number of gaming hours, and Kruskal Wallis test revealed significantly higher scores of the IGDS9-SF among those with daily gaming for more than three hours $(\mathrm{H}(2)=88.8, \mathrm{p}=0.001)$. The IGDS9-SF did not correlate with the number of sleep hours $(r=-0.051, p=0.360)$, but it was negatively correlated with sleep quality $(\mathrm{r}=-0.120, \mathrm{p}=0.05)$. It negatively correlated with the single measure of self-esteem, albeit non-significantly.

The IGDS9-SF strongly correlated with Gender. Mann Whitney U test revealed significantly higher IGD level among males than females $(U=9261.0, z=-4.21, p=0.001)$. Males recorded more daily time ( $>3$ hours) spent in gaming than females $(\chi 2(1)=32.19$, $\mathrm{p}=0.001)$. Mann Whitney $U$ test also revealed significantly higher scores of Social, Escape, Coping, Fantasy, and Recreation motives among males than females ( $\mathrm{U}=9724.0,10934.5$, 10987.0, 10619.0, 8382.0; $\mathrm{z}=-3.66,-2.19,-2.12,-2.59,-5.29 ; \mathrm{p}=0.001,0.028,0.034,0.010$, 0.001). IGD correlated with ethnicity, and Mann Whitney U test revealed significantly higher IGD among Sinhala students compared with students from other ethnicities (U $=7446.0, \mathrm{z}=-2.35, \mathrm{p}=0.019)$.

Despite tendency toward non-invariance across income groups, no significant between group differences in the scores of the IGDS9-SF were noted among income groups $(\mathrm{H}(2)=0.72, \mathrm{p}=0.696)$. Significant differences in IGD were recorded among game types, with the lowest scores reported among single offline players and the highest scores expressed among online multiplayers $(\mathrm{H}(2)=9.70, \mathrm{p}=0.008)$. Multiple online players $(28.0 \%)$ spent more than three hours gaming a day compared with $11.0 \%$ of offline players and $2.0 \%$ of single online players $(\chi 2(4)=41.54, \mathrm{p}=0.001)$. They also exhibited significantly higher levels of Social, Competition, Skill development, and Recreation motives $(\mathrm{H}$ $(2)=47.49,20.06,11.83,18.99$; all $p$ values $=0.001)$.

\section{Discussion}

Concerns about psychometric equivalence of IGD measures in different parts of the world represent a challenge for adequate identification of IGD in different cultural contexts [24]. The psychometric properties of the IGDS9-SF have been largely tested in English-speaking, European, and a small number of less developed countries. Accordingly, the current study complements existing knowledge by examining the psychometric properties of this scale among respondents from Sri Lanka and evaluating its measurements invariance across four international groups from distinct cultural backgrounds.

Consistent with previous studies, data obtained from the Sri Lankan and international samples expressed good fit of the single factor structure of the IGDS9-SF, with all items adequately loading on this factor (Table 2, Figure 2). Multigroup CFA revealed noninvariance of this measure at the configural, metric, and scalar levels across the international participants as well as across various groups in the Sri Lanka sample. However, the IGDS9-SF did not hold strict invariance in the international sample, and there was a tendency toward strict non-invariance across groups of gender and game type in the Sri Lankan sample. Obviously, slight improvements in the fit of the IGDS9-SF were attained by correlating the error terms of item 7 (deceiving others) and item 9 (jeopardizing relationships) in the Turkish sample. Among Turkish adolescents, excessive social media use is associated with an interplay between family life satisfaction and social connectedness [35]. Therefore, the interaction between items of deception and jeopardizing relationships 
may reflect on a subtle relation factor underlying IGD in the Turkish context. On the other hand, correlating the error of item 5 (loss of interest) with item 8 (escape) and the errors of item 2 (withdrawal-Irritability when reducing or stopping use) and item 3 (tolerance - need to spend more time gaming) improved the fit in the Australian and American samples. It seems that gaming is adopted as a measure to escape negative emotions (loss of interest) in the Australian and American contexts. Meanwhile, the mood-modifying effect that results from achieving status or progress in online gaming diminishes in persons with prolonged engagement [36]. Therefore, loss of interest in previous gaming activities may derive tolerance-gaming for a longer time to achieve the previous satisfactory effect. Accordingly, lack of satisfaction of gamers' emotional needs may be related to irritability upon reducing/stopping (withdrawal). Cumulative knowledge shows variations in the internal and external events, which shape the psychosocial background of IGD among individuals meeting the diagnostic criteria of IGD [37]. Thus, further investigations are needed to explore different dynamics underlying IGD in the cultures addressed in our study and whether they affect people's responses to the items of the scale.

Although the loadings of items 2 and 3 were strong in our American and Australian samples, they contributed to metric non-invariance in Australian respondents in a previous assessment of time invariance across Australian and American gamers. In the same Australian sample, items 4, 6, 8, and 9 were involved in partial scalar non-invariance of the scale [24]. Likewise, the single IGD factor was replicated in Polish gamers; however, poor fit was expressed by item 6 (continuation), item 7, and item 8 [17]. In another study comprising participants from the UK, USA, and India, cross-country variations were noted for items pertaining to preoccupation, tolerance, deception, escape, impairment in daily activities, and lack of control [21]. These findings generally suggest that the IGDS9SF possibly involves a general component of high time and energy investment into IGD, in addition to the specific core components of IGD [38,39].

Configural and metric invariance of the IGDS9-SF across countries in our study indicates that IGD is similarly conceptualized by participants from poor countries (e.g., Sri Lanka), countries with evolving economies (e.g., Turkey), and developed countries (Australia and the USA). Strict non-invariance is rarely achieved while one third of the commonly used psychometric measures exhibit partial non-invariance [22,40]. Because the scale demonstrated scalar invariance across countries, the findings of this study show that the IGDS9-SF can be reliably used to compare IGD levels in those countries [40]. Significant differences in the level of IGD were noted among the international groups, with the highest occurrence reported among participants from the USA and Australia. On the contrary, Turkish students reported the lowest level followed by Sri Lankan students. In accordance, aggregate date show that the levels of IGD and social media abuse are evidently higher in Europe and America than Asia [41]. These findings lend further support to previous studies reporting more pathologized IGD scores less close to the mean among respondents from individualistic cultural orientations such as the USA and close to normal scores among collectivistic or less individualistic countries (e.g., Turkey and the UK) $[10,21]$. Noteworthily, the IGDS9-SF was examined in India before. However, it was administered in English [21], with a possibility that the scale may demonstrate different properties if it was administered among Indians who speak only local languages. In the present study, the IGD-SF9 was presented both in English and Sinhala, and it maintained perfect invariance across languages used, with no significant IGD differences between Sinhala and respondents from other ethnicities. Given the proximity as well as the cultural and geographical similarities between India and Sri Lanka, the IGDS9-SF seems to be a suitable measure in this region of south Asia.

Non-invariance of the IGDS9-SF across different groups (genders, ethnicities, game types, etc.) denote that the IGDS9-SF operates evenly as an IGD measure among youth from different social backgrounds in Sri Lanka. However, IGD significantly correlated with gender, with males expressing significantly higher IGD levels, IGD motives, and gaming time than females. This finding is consistent with reports of a recent meta-analysis 
comprising studies across 22 countries, which confirms that men exhibit significantly higher levels of IGD than women. On the other hand, women express higher levels of excessive social media abuse than men [41]. Increased risk for IGD among males is likely to be attributed to gender differences in the activation and connectivity of brain regions associated with the mesocorticolimbic reward system [37]. While females retreat so social networking to meet their need to relate [39], it seems that males retreat to gaming to satisfy their need to relate as noted by significantly higher levels of Social motives among males in the present study. Moreover, online multiplayers were a majority in this study (Table 1); they also expressed the greatest time spent gaming, as well as the highest levels of IGD, and gaming motives (Social, Competition, Skill development, and Recreation), particularly than offline single players. A qualitative investigation shows that distinctions between online and offline gaming can be largely shaped by the development of relationships, norms, and expectations - a person frequently plays with 'people like me' [42]. Overall, males engage more in gaming than females, engage more in online multi-playing, and endorse more Social and Competition motives, denoting that gaming is probably employed to meet social needs.

\section{Conclusions}

The IGDS9-SF expressed good fit and invariance across different groups, along with satisfactory levels of reliability and criterion validity in Sri Lankan students. Males and online multiplayers spent more time playing and expressed higher levels of IGD and playing motives. Significantly higher levels of the social dimension of the MOGQ in both groups suggest that gaming is used to meet the need to relate. Future investigations of relevant factors (e.g., family relations, social connectedness, social skills/competence) may provide further explanations of the use of gaming by males to satisfy their social needs.

In addition to demonstrating good fit of the unidimensional structure among the Turkish, Australian, and American subsamples, the IGDS9-SF also expressed configural, metric, and scalar invariance across countries. Therefore, this scale may be reliably used to compare IGD levels in those countries. The covariances of error terms of items related to deceiving others and jeopardizing relationships in the Turkish sample suggest the involvement of social factors in IGD in this culture. On the other hand, covariances between the error terms of items 5 and 8 as well as items 2 and 3 in the Australian and American subsamples indicate that gaming is employed by respondents from those countries to escape negative emotions. However, failure of gaming to resolve negative emotions may contribute to continued gaming (tolerance) and withdrawal symptoms upon sudden cessation. Future studies examining cultural invariance of the IGDS9-SF may need to identify the dynamics underling IGD in different countries and whether they may affect individuals' response to the items of the IGDS9-SF.

Supplementary Materials: The following are available online at www.mdpi.com/xxx/s1, Table S1: Invariance of IGDS9-SF across different student groups in the Sri Lankan sample.

Author Contributions: Conceptualization, A.M.A., A.O.H. and R.A.; methodology, A.M.A., S.A. and A.M.H.M., H.K.; software A.M.A. and T.S.A.; validation, A.M.A., A.O.H. and R.A.; formal analysis, A.M.A., M.A. and A.O.H.; resources, S.A., M.A. and A.A.A.; data curation, S.A. and T.S.A.; writing-original draft preparation, A.M.H.M., H.K., and A.A.A.; writing-review and editing, A.M.A. and R.A.; project administration, A.M.A. All authors have read and agreed to the published version of the manuscript.

Funding: This research received no external funding.

Institutional Review Board Statement: This study is based on publicly accessible dataset. Therefore, ethical approval is waived.

Informed Consent Statement: Not applicable.

Data Availability Statement: The dataset used to produce the current article are publicly available at: https://data.mendeley.com/datasets/8r2jgm6ygh/2 [26] (access on 2 August 2021). 
Acknowledgments: The authors would like to thank the Deanship of Scientific Research, Qassim University, for funding the publication of this paper.

Conflicts of Interest: The authors declare no conflict of interest.

\section{References}

1. Dong, H.; Yang, F.; Lu, X.; Hao, W. Internet Addiction and Related Psychological Factors Among Children and Adolescents in China During the Coronavirus Disease 2019 (COVID-19) Epidemic. Front Psychiatry 2020, 11, 00751, doi:10.3389/fpsyt.2020.00751.

2. Amin, K.P.; Griffiths, M.D.; Dsouza, D.D. Online Gaming During the COVID-19 Pandemic in India: Strategies for WorkLife Balance. International journal of mental health and addiction 2020, 10.1007/s11469-020-00358-1, doi:10.1007/s11469-02000358-1.

3. Paschke, K.; Austermann, M.I.; Simon-Kutscher, K.; Thomasius, R. Adolescent gaming and social media usage before and during the COVID-19 pandemic. SUCHT 2021, 67, 13-22, doi:10.1024/0939-5911/a000694.

4. Xu, S.; Park, M.; Kang, U.G.; Choi, J.S.; Koo, J.W. Problematic Use of Alcohol and Online Gaming as Coping Strategies During the COVID-19 Pandemic: A Mini Review. Front Psychiatry 2021, 12, 685964, doi:10.3389/fpsyt.2021.685964.

5. Lemenager, T.; Neissner, M.; Koopmann, A.; Reinhard, I.; Georgiadou, E.; Müller, A.; Kiefer, F.; Hillemacher, T. COVID-19 Lockdown Restrictions and Online Media Consumption in Germany. Int. J. Environ. Res. Public Health 2020, 18, doi:10.3390/ijerph18010014.

6. Ali, A.M.; Alkhamees, A.A.; Elhay, E.S.A.; Taha, S.M.; Hendawy, A.O. COVID-19-related psychological trauma and psychological distress among community-dwelling psychiatric patients: people struck by depression and sleep disorders endure the greatest burden Frontiers in public health 2022, 10.3389/fpubh.2021.799812, doi:10.3389/fpubh.2021.799812.

7. Gomez, R.; Stavropoulos, V.; Beard, C.; Pontes, H.M. Item Response Theory Analysis of the Recoded Internet Gaming Disorder Scale-Short-Form (IGDS9-SF). International journal of mental health and addiction 2019, 17, 859-879, doi:10.1007/s11469-018-9890-z.

8. Stavropoulos, V.; Beard, C.; Griffiths, M.D.; Buleigh, T.; Gomez, R.; Pontes, H.M. Measurement Invariance of the Internet Gaming Disorder Scale-Short-Form (IGDS9-SF) Between Australia, the USA, and the UK. International journal of mental health and addiction 2018, 16, 377-392, doi:10.1007/s11469-017-9786-3.

9. Bernaldo-de-Quirós, M.; Labrador-Méndez, M.; Sánchez-Iglesias, I.; Labrador, F.J. Measurement instruments of online gaming disorder in adolescents and young people according to DSM-5 criteria: a systematic review. Adicciones 2020, 32, 291302, doi:10.20882/adicciones.1277.

10. Evren, C.; Dalbudak, E.; Topcu, M.; Kutlu, N.; Evren, B.; Pontes, H.M. Psychometric validation of the Turkish nine-item Internet Gaming Disorder Scale-Short Form (IGDS9-SF). Psychiatry research 2018, 265, 349-354, doi:10.1016/j.psychres.2018.05.002.

11. Zhu, S.; Zhuang, Y.; Lee, P.; Li, J.C.; Wong, P.W.C. Leisure and Problem Gaming Behaviors Among Children and Adolescents During School Closures Caused by COVID-19 in Hong Kong: Quantitative Cross-sectional Survey Study. JMIR serious games 2021, 9, e26808, doi:10.2196/26808.

12. Mamun, M.A.; Ullah, I.; Usman, N.; Griffiths, M.D. PUBG-related suicides during the COVID-19 pandemic: Three cases from Pakistan. Perspect. Psychiatr. Care 2020, 10.1111/ppc.12640, doi:10.1111/ppc.12640.

13. Wu, A.M.; Lai, M.H.; Yu, S.; Lau, J.T.; Lei, M.W. Motives for online gaming questionnaire: Its psychometric properties and correlation with Internet gaming disorder symptoms among Chinese people. J Behav Addict 2017, 6, 11-20, doi:10.1556/2006.6.2017.007. 
14. Beranuy, M.; Machimbarrena, J.M.; Vega-Osés, M.A.; Carbonell, X.; Griffiths, M.D.; Pontes, H.M.; González-Cabrera, J. Spanish Validation of the Internet Gaming Disorder Scale-Short Form (IGDS9-SF): Prevalence and Relationship with Online Gambling and Quality of Life. International Journal of Environmental Research and Public Health 2020, 17, 1562.

15. Kim, B.N.; Ko, H. Psychometric Properties of the Nine-Item Korean Internet Gaming Disorder Scale: Short Form. Cyberpsychology, behavior and social networking 2020, 23, 854-859, doi:10.1089/cyber.2020.0227.

16. Lei, W.; Liu, K.; Zeng, Z.; Liang, X.; Huang, C.; Gong, K.; He, W.; Xiang, B.; Zhang, J.; Zheng, X., et al. The psychometric properties of the Chinese version internet gaming disorder scale. Addict. Behav. 2020, 106, 106392, doi:10.1016/j.addbeh.2020.106392.

17. Schivinski, B.; Brzozowska-Woś, M.; Buchanan, E.M.; Griffiths, M.D.; Pontes, H.M. Psychometric assessment of the Internet Gaming Disorder diagnostic criteria: An Item Response Theory study. Addict Behav Rep 2018, 8, 176-184, doi:10.1016/j.abrep.2018.06.004.

18. Hawi, N.S.; Samaha, M. Validation of the Arabic Version of the Internet Gaming Disorder-20 Test. Cyberpsychology, behavior and social networking 2017, 20, 268-272, doi:10.1089/cyber.2016.0493.

19. Pontes, H.M.; Griffiths, M.D. Portuguese Validation of the Internet Gaming Disorder Scale-Short-Form. Cyberpsychology, behavior and social networking 2016, 19, 288-293, doi:10.1089/cyber.2015.0605.

20. Ali, A.M.; Hori, H.; Kim, Y.; Kunugi, H. The Depression Anxiety Stress Scale 8-items expresses robust psychometric properties as an ideal shorter version of the Depression Anxiety Stress Scale 21 among healthy respondents from three continents. Front. Psychol. 2022, accepted manuscript.

21. Pontes, H.M.; Stavropoulos, V.; Griffiths, M.D. Measurement Invariance of the Internet Gaming Disorder Scale-Short-Form (IGDS9-SF) between the United States of America, India and the United Kingdom. Psychiatry research 2017, 257, 472-478, doi:10.1016/j.psychres.2017.08.013.

22. Ali, A.M.; Hendawy, A.O.; Ahmad, O.; Sabbah, H.A.; Smail, L.; Kunugi, H. The Arabic version of the Cohen perceived stress scale: factorial validity and measurement invariance. Brain Sciences 2021, 11, 419, doi:https://doi.org/10.3390/brainsci11040419.

23. Ali, A.M.; Alkhamees, A.A.; Hori, H.; Kim, Y.; Kunugi, H. The Depression Anxiety Stress Scale 21: Development and Validation of the Depression Anxiety Stress Scale 8-item in Psychiatric Patients and the General Public for Easier Mental Health Measurement in a Post-COVID-19 World. Int. J. Environ. Res. Public Health 2021, 18, 10142, doi:https://doi.org/10.3390/ijerph181910142.

24. Stavropoulos, V.; Bamford, L.; Beard, C.; Gomez, R.; Griffiths, M.D. Test-Retest Measurement Invariance of the Nine-Item Internet Gaming Disorder Scale in Two Countries: A Preliminary Longitudinal Study. International journal of mental health and addiction 2019, 10.1007/s11469-019-00099-w, doi:10.1007/s11469-019-00099-w.

25. Ali, A.M.; Hendawy, A.O.; Almarwani, A.M.; Alzahrani, N.; Ibrahim, N.; Alkhamees, A.A.; Kunugi, H. The Six-item Version of the Internet Addiction Test: Its development, psychometric properties, and measurement invariance among women with eating disorders and healthy school and university students. Int J Environ Res Public Health 2021, 18, 12341, doi:https://doi.org/10.3390/ijerph182312341.

26. Manchanayake, M. Data For: Internet Gaming Disorder: An Emerging Public Health Concern Among an Advanced Level Student Population from Colombo, Sri Lanka. Mendeley Data 2021, Version 1, doi:10.17632/8r2jgm6ygh.1.

27. Manchanayake, M.; Malsirini, T.G.M.; Vithanage, M.; Jayawardene, D.M.S. Internet Gaming Disorder: Prevalence and Correlates Among an Advanced Level Student Population from the Colombo Educational Zone. In Proceedings of 26th Annual Academic Sessions of the College of Community Physicians, Sri Lanka. 
28. Demirbaş-Çelik, N.; Yeşilyurt, F.; Çelik, B. The Mediating Role Of Life Satisfaction, Internet Gaming Disorder, And Negative Affect Within The Relationship Of Basic Psychological Needs Satisfaction And Depression. Mendeley Data 2021, V3, doi:10.17632/k698sznwf6.3.

29. Burleigh, T.L. IGD_Culture. Data Archiving and Networked Services (DANS) 2019, V1, doi:10.17026/dans-xgs-hpdn.

30. Andreetta, J.; Teh, M.J.; Burleigh, T.L.; Gomez, R.; Stavropoulos, V. Associations between comorbid stress and Internet Gaming Disorder symptoms: Are there cultural and gender variations? Asia-Pacific psychiatry : official journal of the Pacific Rim College of Psychiatrists 2020, 12, e12387, doi:10.1111/appy.12387.

31. Pontes, H.M.; Griffiths, M.D. Measuring DSM-5 internet gaming disorder: Development and validation of a short psychometric scale. Comput. Human Behav. 2015, 45, 137-143, doi:https://doi.org/10.1016/j.chb.2014.12.006.

32. Demetrovics, Z.; Urbán, R.; Nagygyörgy, K.; Farkas, J.; Zilahy, D.; Mervó, B.; Reindl, A.; Ágoston, C.; Kertész, A.; Harmath, E. Why do you play? The development of the motives for online gaming questionnaire (MOGQ). Behav. Res. Methods 2011, 43, 814-825, doi:10.3758/s13428-011-0091-y.

33. Snyder, E.; Cai, B.; DeMuro, C.; Morrison, M.F.; Ball, W. A New Single-Item Sleep Quality Scale: Results of Psychometric Evaluation in Patients With Chronic Primary Insomnia and Depression. J. Clin. Sleep Med. 2018, 14, 1849-1857, doi:doi:10.5664/jcsm.7478.

34. Ali, A.M.; Hori, H.; Kim, Y.; Kunugi, H. Predictors of nutritional status, depression, internet addiction, Facebook addiction, and tobacco smoking among women with eating disorders in Spain. Frontiers in Psychiatry 2021, 12, 2001, doi:10.3389/fpsyt.2021.735109.

35. Savci, M.; Akat, M.; Ercengiz, M.; Griffiths, M.D.; Aysan, F. Problematic Social Media Use and Social Connectedness in Adolescence: The Mediating and Moderating Role of Family Life Satisfaction. International journal of mental health and addiction 2020, 10.1007/s11469-020-00410-0, doi:10.1007/s11469-020-00410-0.

36. King, D.L.; Herd, M.C.E.; Delfabbro, P.H. Tolerance in Internet gaming disorder: A need for increasing gaming time or something else? J Behav Addict 2017, 6, 525-533, doi:10.1556/2006.6.2017.072.

37. Paulus, F.W.; Ohmann, S.; von Gontard, A.; Popow, C. Internet gaming disorder in children and adolescents: a systematic review. Dev. Med. Child Neurol. 2018, 60, 645-659, doi:10.1111/dmcn.13754.

38. Atroszko, P.A.; Balcerowska, J.M.; Bereznowski, P.; Biernatowska, A.; Pallesen, S.; Schou Andreassen, C. Facebook addiction among Polish undergraduate students: Validity of measurement and relationship with personality and well-being. Comput. Human Behav. 2018, 85, 329-338, doi:https://doi.org/10.1016/j.chb.2018.04.001.

39. Ali, A.M.; Hendawy, A.O.; Elhay, E.S.A.; Ali, E.M.; Alkhamees, A.A.; Kunugi, H.; Hassan, N.I. The Bergen Facebook Addiction Scale: Its psychometric properties and invariance among women with eating disorders. BMC Women's Health 2022, under review.

40. Putnick, D.L.; Bornstein, M.H. Measurement Invariance Conventions and Reporting: The State of the Art and Future Directions for Psychological Research. Developmental review : DR 2016, 41, 71-90, doi:10.1016/j.dr.2016.06.004.

41. Su, W.; Han, X.; Yu, H.; Wu, Y.; Potenza, M.N. Do men become addicted to internet gaming and women to social media? A meta-analysis examining gender-related differences in specific internet addiction. Comput. Human Behav. 2020, 113, 106480, doi:https://doi.org/10.1016/j.chb.2020.106480.

42. Eklund, L. Bridging the online/offline divide: The example of digital gaming. Comput. Human Behav. 2015, 53, 527-535, doi:https://doi.org/10.1016/j.chb.2014.06.018. 